\title{
Material Properties
}

\section{Investigation of the Electrical Properties of XLPE/SiC Nanocomposites}

\author{
Youyuan Wang, Can Wang, Kun Xiao
}

State Key Laboratory of Power Transmission Equipment \& System Security and New Technology

Chongqing University, Chongqing, 400030, China

\begin{abstract}
The interface between nanoparticles and the polymer matrix, which dominates the electrical properties of nanocomposites, can effectively improve the DC breakdown and suppress space charge accumulation in nanocomposites. To research the interface characteristics, XLPE/SiC nanocomposites with concentrations of $1 \mathrm{wt} \%, 3 \mathrm{wt} \%$ and $5 \mathrm{wt} \%$ were prepared. The DC breakdown, dielectric properties and space charge behavior were examined using pulsed electro-acoustic (PEA) equipment and a dielectric analyzer. The test results show that the nanocomposites with concentrations of $1 \mathrm{wt} \%$ and $3 \mathrm{wt} \%$ have higher DC breakdown field strength than neat XLPE. In contrast, there is a lower DC breakdown strength at a concentration of $5 \mathrm{wt} \%$, possibly due to the agglomeration of nanoparticles. Nanoparticle doping increases the real and imaginary permittivities over those of neat XLPE. Furthermore, with increasing concentration, a larger increase in the permittivity amplitude was observed. Based on the space charge behavior, all nanocomposites could suppress space charge accumulation, but the nanocomposite with a concentration of $1 \mathrm{wt} \%$ exhibited the best effect. Meanwhile, heterocharge accumulation near electrodes was observed in neat XLPE and the nanocomposite with a concentration of $5 \mathrm{wt} \%$. In contrast, homocharge accumulation near electrodes was observed in the nanocomposite with a concentration of $3 \mathrm{wt} \%$. This phenomenon may be due to different amounts of shallow traps in nanocomposites with different concentrations, which might lead to differing electron or hole mobility.
\end{abstract}

Keywords: XLPE/SiC nanocomposites, electrical properties, interface, mobility

\section{INTRODUCTION}

Polyethylene is an excellent insulation material used in high-voltage cables. However, under a high-voltage DC field, space charges can accumulate in polyethylene, which can distort the electrical field and cause partial discharge. Furthermore, the process of trapping and de-trapping of electrons or holes is accompanied by the release of energy, leading to the aging of insulation materials[1-4].

In developing DC transmission technology, methods to suppress space charge accumulation and improve the DC breakdown strength of insulation materials are key. Many studies indicate that composites such as polymer/nanoparticles, polymer/zeolite and epoxy/clay can suppress space charge accumulation and improve DC breakdown strength effectively[5-10]. Since T. J. Lewis[11] first proposed the concept of nanodielectrics in 1994, polyethylene-based nanocomposites have developed from LDPE nanocomposites to XLPE nanocomposites. It has been demonstrated that XLPE nanocomposites can improve electrical properties[12-17]. The interface in nanocomposites between the polymer matrix and nanoparticles is the main reason for the improvement in the electrical properties[18-24]. To understand the characteristics of this interface, some microscopic models have 
been proposed, such as the multi-core model[25-26]. However, because the relationship between the nanoparticles and the polymer matrix is unclear, there are some limitations in the existing interface models. Therefore, it is necessary to perform further studies on the mechanism of the interface between nanoparticles and the polymer matrix.

In this paper, XLPE/SiC nanocomposites with different concentrations were prepared. The electrical properties including DC breakdown, dielectric properties and space charge behavior have been tested. The mechanism of improvement of DC breakdown strength and movement of carriers in nanocomposites are discussed, indicating that $\mathrm{SiC}$ nanoparticles can promote the electrical properties of XLPE.

\section{EXPERIMENTAL}

\subsection{Sample Preparation}

The base polymer used was an additive-free low density polyethylene (LDPE) with a melt flow index $2.1 \sim 2.2 \mathrm{~g} / \mathrm{min}$ and a density of $0.910 \sim 0.925 \mathrm{mg} / \mathrm{cm}^{3}$, which was produced by Daqing Petrochemical Co. China. The nanoparticles used were silicon carbide (SiC) with a 40-nm particle size produced by Shanghai Jingchun Scientific Co. To remove moisture, which might affect the electrical properties of nanocomposites, the nanoparticles were dried in a vacuum drier at $190{ }^{\circ} \mathrm{C}$ for $24 \mathrm{~h}$. Then, $1 \mathrm{wt} \%, 3 \mathrm{wt} \%$ and $5 \mathrm{wt} \%$ of SiC nanoparticles were mixed into LDPE in a twin screw extruder. The mixing process was conducted for $20 \mathrm{~min}$ using the circulation mode of the extruder. Considering the dicumyl peroxide (DCP) melting point of approximately $128^{\circ} \mathrm{C}, 2 \mathrm{wt} \%$ of DCP was mixed with the LDPE/SiC nanocomposites at $116^{\circ} \mathrm{C}$. Then, the XLPE/SiC nanocomposites were extruded and the extrudate granulated. Samples with a thickness of approximately $190 \mu \mathrm{m}$ were prepared in a press at $180^{\circ} \mathrm{C}$. To remove any residue, all samples were degassed in a vacuum drier for $1 \mathrm{~h}$ at $80^{\circ} \mathrm{C}$.

2.2 Scanning electron microscope imaging

A sample was fractured in liquid nitrogen and the fracture surface observed by scanning electron microscopy (SEM, MERLIN Compact) to reveal the nanofiller dispersion .

\subsection{DC breakdown}

Samples were placed between spherical electrodes $(\varnothing=6.3 \mathrm{~cm})$, which were immersed in transformer oil to avoid surface flashover on the samples. The applied DC voltage was increased linearly at a rate of $0.5 \mathrm{kV} / \mathrm{s}$ until breakdown occurred. The thickness of the tested samples was measured to calculate the DC breakdown strength. The two-parameter Weibull distribution was used to analyze the DC breakdown characteristics of the samples.

2.4 Dielectric properties

The dielectric properties of the materials were measured in the frequency domain from $10^{-1} \mathrm{~Hz}$ to $10^{6} \mathrm{~Hz}$ at room temperature using a Novocontrol ALPHA-A high resolution dielectric analyzer. Prior to dielectric analysis, gold electrodes were deposited onto both surfaces of the specimens by sputtering. The diameter of the sputtered electrodes was $15 \mathrm{~mm}$.

2.5 Space charge behavior

Space charge measurements were performed using a pulsed electro-acoustic (PEA) system with a pulse width of $2 \sim 5 \mathrm{~ns}$, pulse amplitude of $200 \mathrm{~V}$ and output voltage of $0 \sim 20 \mathrm{kV}$. All samples were measured at room temperature $\left(25+1{ }^{\circ} \mathrm{C}\right)$. A DC electrical field was applied for $1 \mathrm{~h}$, and the space charge formation was confirmed after the polarization times of $300 \mathrm{~s}, 900 \mathrm{~s}$ and $1800 \mathrm{~s}$. 


\section{RESULTS}

\subsection{Scanning electron microscope imaging}

Figure 1 is a SEM image showing the dispersion of nanofiller in the nanocomposites. As shown in this image, the $\mathrm{SiC}$ nanoparticles are well distributed in the nanocomposites with concentration of $1 \mathrm{wt} \%$. At the concentration is $3 \mathrm{wt} \%$, some obvious agglomerations exist in the nanocomposites. When the concentration is $5 \mathrm{wt} \%$, few separated nanoparticles can be observed in the nanocomposites, which means many more nanoparticle agglomerates. These results will directly affect the electrical properties of nanocomposites.

\subsection{DC breakdown}

FIG. 1. SEM section images of XLPE/SiC

The DC breakdown properties of insulation are affected by many factors. Randomness and dispersion of DC breakdown cannot be prevented even under identical conditions. It is recognized that the two-parameter Weibull probability can fit the DC breakdown strength of solid materials effectively with the following expression[27]:

$$
\left.F(U ; \alpha, \beta)=1 \exp \left(\frac{U}{\alpha}\right)^{\beta}\right)
$$

where $U$ represents the DC breakdown strength; $\alpha$ represents scale parameters, indicating the DC breakdown strength when the failure rate is 0.632 ; and $\beta$ represents shape parameters, indicating the randomness of data.

Figure 2 is the Weibull probability plot for the DC breakdown of XLPE and XLPE/SiC nanocomposites, and the scale parameters and shape parameters are listed in table 1.

FIG. 2. Weibull probability plot for the DC breakdown strength of XLPE and XLPE/SiC nanocomposites

Table 1. Scale parameter $\boldsymbol{\alpha}$ and shape parameter $\boldsymbol{\beta}$ for XLPE and XLPE/SiC nanocomposites

\begin{tabular}{ccccc}
\hline & XLPE & $\begin{array}{c}1 \mathrm{wt} \% \\
\text { XLPE/SiC }\end{array}$ & $\begin{array}{c}3 \mathrm{wt} \% \\
\text { XLPE/SiC }\end{array}$ & $\begin{array}{c}5 \mathrm{wt} \% \\
\text { XLPE/SiC }\end{array}$ \\
\hline $\boldsymbol{\alpha}(\mathrm{kV} / \mathrm{mm})$ & 260.67 & 331.99 & 297.19 & 244.71 \\
\hline $\boldsymbol{\beta}$ & 13.08 & 24.72 & 15.97 & 9.71 \\
\hline
\end{tabular}

As shown in table 1, nanocomposites with concentrations of $1 \mathrm{wt} \%$ and $3 \mathrm{wt} \%$ have larger scale parameters than neat XLPE. In contrast, there is a smaller scale parameter in the nanocomposite with a concentration of $5 \mathrm{wt} \%$. This means that the DC breakdown strength of nanocomposites decreases with the increase of concentration of nanoparticles, which may be caused by the distribution of nanoparticles in nanocomposites. More details will be discussed in section 4.1. Additionally, the shape parameter is highest in the nanocomposite with a concentration of $1 \mathrm{wt} \%$, indicating that $\mathrm{SiC}$ nanoparticles may decrease the randomness of the DC breakdown data.

3.3 Dielectric properties 
Figure 3 shows the frequency dependence of the real and imaginary permittivities. As shown in 3(a), the real permittivity increases almost linearly with decreasing frequency. Nanoparticle doping clearly increases real permittivity. Meanwhile, with increasing concentration, higher real permittivity was observed in nanocomposites. Furthermore, 3(b) shows that, with decreasing frequency, imaginary permittivity increases in all samples. Slopes are observed in nanocomposites in the frequency domain from $10^{-1}-10^{0}$ and $10^{2}-10^{3}$. Additionally, there are higher increasing amplitudes in the nanocomposites than in neat XLPE.

As we know, the dielectric relaxation of materials is revealed by real and imaginary permittivities. The large amount of intrinsic dipoles distributed in nanocomposites contribute to the real permittivity, in which the rotation is associated with thermal molecular motion. Furthermore, relaxation time is dependent on the stress on molecules, and the time spent during the relaxation process is approximately several nano- or milli-seconds. Additionally, metastable status exists in the intertransformation of a material in which this intertransformation must conquer a potential barrier. Therefore, the time spent may be longer, such as several seconds or hours or years.

In XLPE/SiC nanocomposites, the polymer matrix, nanofillers and interface area have different electric properties, so the polarizations that occur in these three parts are also different. Because of Maxwell-Wagner interface polarization[30], there is a larger permittivity in nanocomposites than in neat XLPE. Furthermore, the real permittivity of nano-SiC is larger than the real permittivity for neat XLPE[31]. Thus, the real permittivity of nanocomposites is higher than that of neat XLPE. In addition, according to the research, there are Gouy-Chapman layers in nanocomposites, in which electrons or holes can move more easily. Therefore, space charge polarization may exist in the interface area at low frequency, increasing the amplitude of the imaginary permittivity in XLPE/SiC nanocomposites.

\section{FIG. 3. Permittivity of neat XLPE and XLPE/SiC nanocomposites}

\subsection{Space charge behavior}

Figure 4 shows the space charge behavior of XLPE and the XLPE/SiC nanocomposites. It is clear that heterocharges accumulate near the electrodes in XLPE. The amount of space charges near the anode electrode is $6.46 \mathrm{C} / \mathrm{m}^{3}$, which is larger than the value of $0.52 \mathrm{C} / \mathrm{m}^{3}$ near the cathode electrode. With increasing polarization time, the amount of space charges becomes less near the anode electrode but more near the cathode electrode.

Comparing the space charge behavior of all samples, $\mathrm{SiC}$ nanoparticles were able to suppress space charge accumulation effectively, especially in the nanocomposite at a concentration of $1 \mathrm{wt} \%$. With increasing concentration, the inhibitory effect of $\mathrm{SiC}$ nanoparticles on space charge accumulation in the nanocomposites gradually decreased. When the concentration was $3 \mathrm{wt} \%$, homocharge accumulation near the electrodes was observed. In contrast, heterocharge accumulation was observed near the electrodes in nanocomposites with a concentration of $5 \mathrm{wt} \%$. In addition, with increasing polarization time, the heterocharges near the anode electrode first decreased and then increased, which is in contrast to the heterocharges near the cathode electrode, which first increased and then gave way to homocharge accumulation. 
FIG. 4. Space charge behavior of XLPE and XLPE/SiC nanocomposite 3.5 Mean volume charge density

To understand the effect of $\mathrm{SiC}$ nanoparticles on the space charge behavior of nanocomposites, the mean volume charge density of nanocomposites was calculated. The expression is as follows[28]:

$$
q\left(t ; E_{p}\right)=\frac{1}{x_{1}-x_{0}} \int_{x_{0}}^{x_{1}}\left|q_{p}\left(x, t ; E_{p}\right)\right| d x
$$

where $x_{0}$ and $x_{1}$ are the positions of the anode and cathode electrodes, respectively (induced charges at the electrodes are not taken into account), $t$ is the depolarization time, $E_{\mathrm{P}}$ is the polarization voltage, and $q_{\mathrm{P}}\left(x, t ; E_{\mathrm{P}}\right)$ is the charge density in the nanocomposites.

Figure 5 shows the mean volume charge density of the neat XLPE and XLPE nanocomposites. According to this image, there are fewer space charges in nanocomposites compared with neat XLPE. In addition, the nanocomposite with a concentration of $1 \mathrm{wt} \%$ has the least amount of space charges in all samples, with a mean volume charge density of $0.20 \mathrm{C} / \mathrm{m}^{3}$ for a polarization time of $900 \mathrm{~s}$. Because the accumulation of electrons and holes came from the electrodes, the mean volume charge density is 1.05 $\mathrm{C} / \mathrm{m}^{3}$ for a polarization time of $300 \mathrm{~s}$ in neat XLPE. With increasing polarization time, heterocharges near the electrodes in neat XLPE are neutralized by electrons or holes from the electrodes. Therefore, the mean volume charge density of neat XLPE decreases with increasing polarization time. Because electrons and holes from the electrodes neutralize the heterocharges near the electrodes in the nanocomposite with a concentration of $5 \mathrm{wt} \%$, the mean volume charge density decreases first with increasing polarization time. When electrons from the cathode accumulate near the anode, the mean volume charge density increases, and homocharges appear near the cathode. Therefore, the mean volume charge density of the nanocomposite with a concentration of $5 \mathrm{wt} \%$ first decreases and then increases with increasing polarization time.

FIG. 5. Mean volume charge density of XLPE and XLPE/SiC nanocomposites

\section{DISCUSSION}

\subsection{Influence of $\mathrm{SiC}$ nanoparticles on DC breakdown strength}

According to the test results, in comparison with neat XLPE, higher DC breakdown strengths were observed in nanocomposites with low concentrations, such as $1 \mathrm{wt} \%$ or $3 \mathrm{wt} \%$. In contrast, there was a low DC breakdown strength for the concentration of $5 \mathrm{wt} \%$. Space charge accumulation occurs when polymer-based nanocomposites are subjected to a DC field, which can affect the amount of electrons or holes injected by electrodes in nanocomposites. Electrons or holes move from electrodes into samples by Schottky emission, and the formula is as follows[29]:

$$
j_{i n}=A T^{2} \exp \left(-\frac{e \phi}{k T}\right) \exp \left(\frac{e}{k T} \sqrt{\frac{e E(0, t)}{4 \pi \varepsilon_{r} \varepsilon_{0}}}\right)
$$

where $j_{\text {in }}$ is the injection current density, $A$ is constant, $\varphi$ is the potential barrier for electron injection, and $E(0, t)$ is the interface electric field.

It is clear that the interface electric field will increase while there are heterocharges near the electrodes in the nanocomposites, which means the $E(0, t)$ in formula (3) will increase at the same time, 
leading to the increase of the amount of electrons or holes injected by electrodes. In contrast, homocharges near the electrodes decrease the amount of electrons or holes in the nanocomposites. Furthermore, the probability of avalanche breakdown is related to the amount of electrons or holes and the mean free path in insulation; thus, there is a high probability of avalanche breakdown in materials where heterocharges are observed near the electrodes. Therefore, as shown in fig 4(a) and (d), heterocharges near electrodes are observed in neat XLPE and nanocomposite with a concentration of 5 $\mathrm{wt} \%$, which may be the reason for the low DC breakdown strength in comparison to nanocomposites with concentrations of $1 \mathrm{wt} \%$ and $3 \mathrm{wt} \%$.

FIG. 6. Debye shielding layer of nanoparticle

In addition, according to the multi-core model proposed by $\mathrm{T}$. Tanaka[26], electrons may lose energy when moving into the Gouy-Chapman diffuse layer and then organizing around nanoparticles, as shown in fig 6 . When the nanocomposites have low concentration of nanoparticles, such as 1 wt $\%$ and $3 \mathrm{wt} \%$, the Gouy-Chapman diffuse layers may not overlap because of the large distance between nanoparticles, which may decrease the energy of moving electrons and the free electron path effectively. This may be the reason why for the concentrations of $1 \%$ and $3 \mathrm{wt} \%$ the DC breakdown is higher than that for the case of the sample with the concentration of $5 \mathrm{wt} \%$. However, when the concentration is high, such as at $5 \mathrm{wt} \%$, it is difficult to make the nanoparticles disperse well in the polymer matrix. Therefore, agglomerates occur in nanocomposites, decreasing the specific surface area of the nanoparticles and possibly affecting the characteristics of the Gouy-Chapman diffuse layer. Additionally, overlaps of the Gouy-Chapman diffuse layer may exist with increasing concentration.

\subsection{Influence of $\mathrm{SiC}$ nanoparticles on space charge behavior}

Space charge accumulation and movement occur in polyethylene under DC voltage. These electrons or holes are mainly from the electrodes or from impurities that decompose under high voltage. Because of the existence of defects and dipoles in polyethylene, there are charge traps with different depths, which can trap electrons or holes. When the amount of trapped electrons or holes is large, there is a space charge accumulation in polyethylene. In addition, electrons or holes may de-trap from shallow traps because of an electrical field force or thermal movement. Meanwhile, the de-trapped electrons or holes will move to another electrode. Therefore, the electrons or holes move to another electrode in a trapping and de-trapping pattern. When the electrons or holes are trapped, space charge accumulation occurs in polyethylene. Furthermore, considering a large amount of charge traps with different depths that are distributed in polymer, nanoparticle doping may decrease the amount of nano-defects and the depth of charge traps. However, because of the large special surface area of nanoparticles, the interface between the nanoparticles and the polymer matrix might increase the number of shallow and deep charge traps. Thus, space charge behavior in nanocomposites may be affected by the concentration of nanoparticles.

Currently, some researchers think that heterocharges near electrodes in nanocomposites are caused by impurities that may decompose under a high DC field. Other researchers think that the electrons or holes originate from an electrode and then move to another electrode with a tendency to accumulate near electrodes, leading to heterocharges. According to our research, there are heterocharges near the electrodes in both XLPE and XLPE/SiC nanocomposite with a concentration of $5 \mathrm{wt} \%$, as shown in 
Figure 4(a) and (b). Additionally, the amount of heterocharges decreases with increasing polarization time. Therefore, the heterocharges observed in this study can be explained by the above two cases. At the initial stage of polarization, impurities decompose under a high DC field producing heterocharges in the nanocomposites. With increasing polarization time, the amount of electrons or holes from electrodes increases, which may neutralize the heterocharges near the electrodes. This phenomenon especially occurs in nanocomposites with a concentration of $5 \mathrm{wt} \%$, in which the heterocharges near the cathode turn into homocharges.

The test results show the least amount of space charge accumulation in nanocomposites with a concentration of $1 \mathrm{wt} \%$. When the concentration is low, such as at $1 \mathrm{wt} \%$, the distance between nanoparticles is large, which means that each nanoparticle can be regarded as an independent individual. According to the research on LDPE/MgO by T. Tanaka[32], there are deep traps surrounding $\mathrm{MgO}$ nanoparticles in nanocomposites, which can attract a number of electrons or holes near $\mathrm{MgO}$ nanoparticles. A single nanoparticle surrounded by electrons or holes represents an electrical neutral. Meanwhile, because nanoparticles are dispersed throughout the whole nanocomposite, there is almost no space charge accumulation in nanocomposites, as shown in Figure 4(b). With increasing concentration, the distances between nanoparticles decrease, causing overlaps of interfaces, which may affect space charge trap distribution and depth. Therefore, more space charge accumulation occurs in nanocomposites with concentrations of $3 \mathrm{wt} \%$ and $5 \mathrm{wt} \%$.

FIG. 7. The motion of electrons and holes in loose layers of nanoparticles

As shown in Figure 4 (c) and (d), homocharges near the electrodes are observed in nanocomposites with a concentration of $3 \mathrm{wt} \%$. In contrast, there are heterocharges near the electrodes in nanocomposites with a concentration of $5 \mathrm{wt} \%$. It is recognized that the movement of electrons or holes may be affected by shallow traps. In short, the conductivity of nanocomposites may be related to the amount of shallow traps. According to the multi-core model promoted by T. Tanaka[26], the interface between the nanoparticles and the polymer matrix can be divided into three layers: the bonded layer, the bound layer and the loose layer. Deep traps induced by this interface may exist in the bonded layer, and shallow traps may exist in the loose layer. Because the thickness of the loose layer may be tens of nanometers, the loose layers may overlap when the concentration is $3 \mathrm{wt} \%$, which can provide access for the movement of electrons or holes. However, because of the limited amount of overlapped nanoparticles, electrons or holes may be trapped after moving some distance, which may cause homocharges near the electrodes in the nanocomposites. Therefore, in the first stage of polarization, there are heterocharges near the electrodes in nanocomposites with a concentration of $3 \mathrm{wt} \%$ because of the decomposition of impurities under high voltage. With increasing polarization time, more electrons or holes from electrodes move in the loose layer, which may cause homocharges in nanocomposites when electrons or holes are trapped. At a concentration of $5 \mathrm{wt} \%$, there are more overlaps of the loose layer because of the large amount of nanoparticles, which will probably provide access between the anode and cathode. Therefore, electrons or holes may move from one electrode to another, leading to heterocharges in the nanocomposites, as shown in Figure 4(d).

5. CONCLUSIONS

The DC breakdown, permittivity and space charge behavior of XLPE/SiC nanocomposites have 
been researched in this paper, and the movement mechanism of electrons and holes has been discussed. The main conclusions are as follows:

(1) Nanocomposites with concentrations of $1 \mathrm{wt} \%$ and $3 \mathrm{wt} \%$ can improve DC breakdown strength effectively. Nanocomposites with a concentration of $1 \mathrm{wt} \%$ have the highest DC breakdown strength with a scale parameter of $331.90 \mathrm{kV} / \mathrm{mm}$.

(2) Because of the Maxwell-Wagner interface polarization and the high permittivity of SiC nanoparticles, the real and imaginary permittivities of nanocomposites are increased in comparison with those of neat XLPE. Furthermore, with increasing concentration, a higher increase in amplitude is observed in the nanocomposites.

(3) Space charge accumulation can be suppressed effectively in nanocomposites by adding SiC nanoparticles. In all samples, nanocomposites with a concentration of $1 \mathrm{wt} \%$ show the least amount of space charges.

(4) When the concentration is low, the distances between nanoparticles are large, which means that each nanoparticle can be regarded as an independent individual. Therefore, low-concentration nanocomposites, such as $1 \mathrm{wt} \%$, have lesser amounts of space charges. With increasing concentration, nanoparticles may agglomerate into nanocomposites, which may affect the interface characteristics between the nanoparticles and the polymer matrix. Therefore, there are more space charges in high-concentration nanocomposites, such as $5 \mathrm{wt} \%$.

\section{ACKNOWLEDGMENT}

This work was supported by State Key Laboratory of Power Transmission Equipment \& System Security and New Technology of China (2007DA10512713205) and the National Science Foundation of China (51321063).

\section{Reference}

[1] R. Kochetov, I. A. Tsekmes, and P. H. F. Morshuis, Smart Mater. Struct., 24, 075019 (2015).

[2] G. Blake, J. Appl. Phys., 77, 2916 (1995).

[3] F. Tian, Q Lei, X. Wang, and Y. Wang, Appl. Phys. Lett., 99, 142903 (2011).

[4] B. Han, X. Wang, Z. Sun, J. Yang, and Q. Lei, Appl. Phys. Lett., 102, 012902 (2013).

[5] M. Sangermano, F. Deorsola, D. Fabiani, G. Montanari,and G. Rizza, J. Appl. Polym. Sci., 114, 2541 (2009).

[6] A.S. Thelakkadan, G. Coletti, F. Guastavino, A. Fina, Polym. Eng. Sci., 52, 1037 (2012).

[7] A.S. Thelakkadan, G. Coletti, F. Guastavino, A. Fina, Polym. Compos., 32, 1499 (2011).

[8] H. Smaouia, L. E.L. Mirc, H. Guermazib, S. Agneld, A. Toureille, J. Alloys Comp, 477, 316 (2009).

[9] X. Huang and P. Jiang, C. U. Kim, J. Appl. Phys., 102, 124103 (2007).

[10] R. Sarathi, R.K. Sahu, P. Rajeshkumar, Mat. Sci. Eng. A-Strict, 445-446, 567 (2007).

[11] T. J. Lewis, “Nanometric dielectric,” IEEE Trans. Dilectr. Electr. Insul., Vol. 1, pp. 812-825, 1994.

[12] J. I. Hong, L. S. Schadler, R. W. Siegel, and E. Ma ${ }^{\circ}$ rtensson, Appl. Phys. Lett., 82, 12 (2003).

[13] Y. Park, J. Kwon, J. Sim, J. Hwang, C. Seo, J. Kim, and K. Lim, Jpn. J. Appl. Phys., 53, 08 NL05 (2014). 
[14]L. Hui, L. S. Schadler and J. K. Nelson, IEEE Trans. Dilectr. Electr. Insul., 20, 641 (2013).

[15] M. Roy, J. K. Nelson, R. K. MacCrone, L. S. Schadler, J. Mater. Sci., 42, 3789 (2007).

[16] J. P. Jose, L. Chazeau, J. Y. Cavaill'e, K. T. Varughesec, and S. Thomas, RSC. Adv., 4, 31643 (2014).

[17]L. Zhang, Y. X. Zhou, M. Huang, Y. C. Sha, J. H. Tian and Q. Ye, IEEE Trans. Dilectr. Electr. Insul., 21, 424 (2014).

[18] T J Lewis, IEEE Trans. Dilectr. Electr. Insul., 11, 739 (2004).

[19]X. Bai, X. He, J. Zhang, X. Zhu, H. Zhang, R. Cheng, B. Liu, Polym. Compos., 33, 940 (2012).

[20] T. Tanaka, IEEE Trans. Dilectr. Electr. Insul., 12, 914 (2005).

[21]M. A. Osman, J. E. P. Rupp, Macromol. Rapid. Comm., 26, 880 (2005).

[22] S. T. Li, G. L. Yin, S. N. Bai and J. Y. Li, IEEE Trans. Dilectr. Electr. Insul., 18, 1535 (2011).

[23] X. Huang, P. Jiang, and Y. Yin, Appl. Phys. Letters., 95, 242905 (2009).

[24]T. J. Lewis, IEEE Trans. Dilectr. Electr. Insul., 21, 497 (2014).

[25]H. Y. Li, G. Liu, B. Liu, W. Chen and S. T. Chen, Materials Letters, 61, 1507 (2007).

[26] T. Tanaka, M. Kozako, N. Fuse and Y. Ohki, IEEE Trans. Dilectr. Electr. Insul., 12, 669 (2001).

[27] M. Jarvid, A. Johansson, R. Kroon, J. M. Bjuggren, H. Wutzel, V. Englund, S. Gubanski, M. R. Andersson and C. Müller, Advanced materials., 27, 897 (2015).

[28] G. Mazzantia, G. C. Montanari, F. Palmieri, and J. Alison, J. Appl. Phys., 94, 5997 (2003).

[29] K. Kaneko, T. Mizutani and Y. Suzuoki, IEEE Trans. Dielectr. Electr. Insul., 6, 152 (1999).

[30]H. Smaouia, L. E.L. Mirc, H. Guermazib, S. Agneld, and A. Toureille, J. Alloy. Compd., 477, 316 (2009).

[31] A. Kassibaa, M. Tabellouta, S. Charpentiera, N. Herlinb, J.R. Emerya, Solid. State. Commun., 115, 389 (2000).

[32] Tatsuo Takada, Yuji Hayase, Yasuhiro Tanaka, and Tatsuki Okamoto, IEEE Trans. Dielectr. Electr. Insul., 15, 152 (2008). 


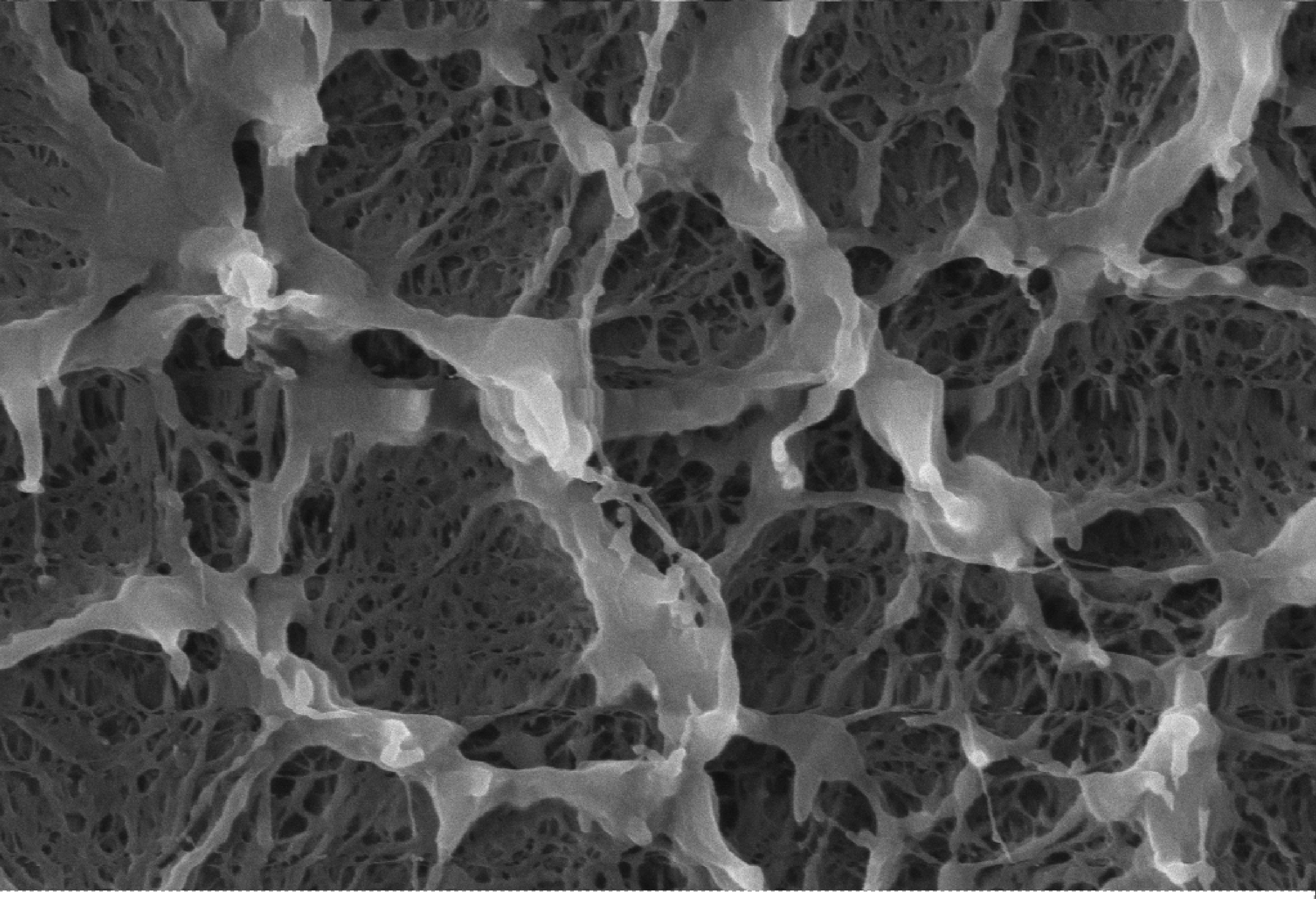

$300 \mathrm{~nm}$

$\mathrm{EHT}=5.00 \mathrm{kV}$ $W D=9.8 \mathrm{~mm}$
Signal $A=$ SE2 Mag $=30.00 \mathrm{KX}$
Date :11 Apr 2014 Time :11:30:40 
Probability plot for Weibull distribution

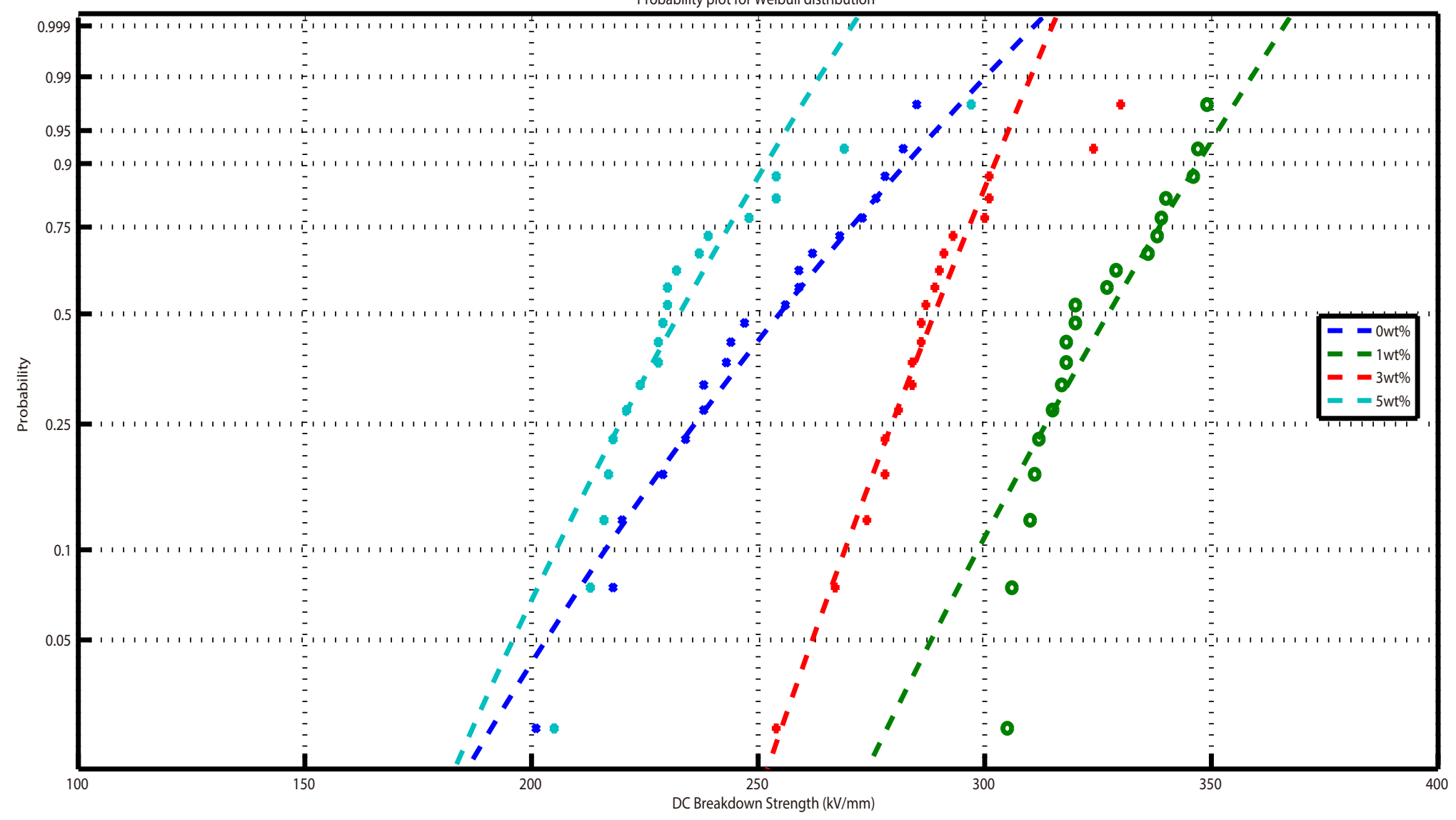




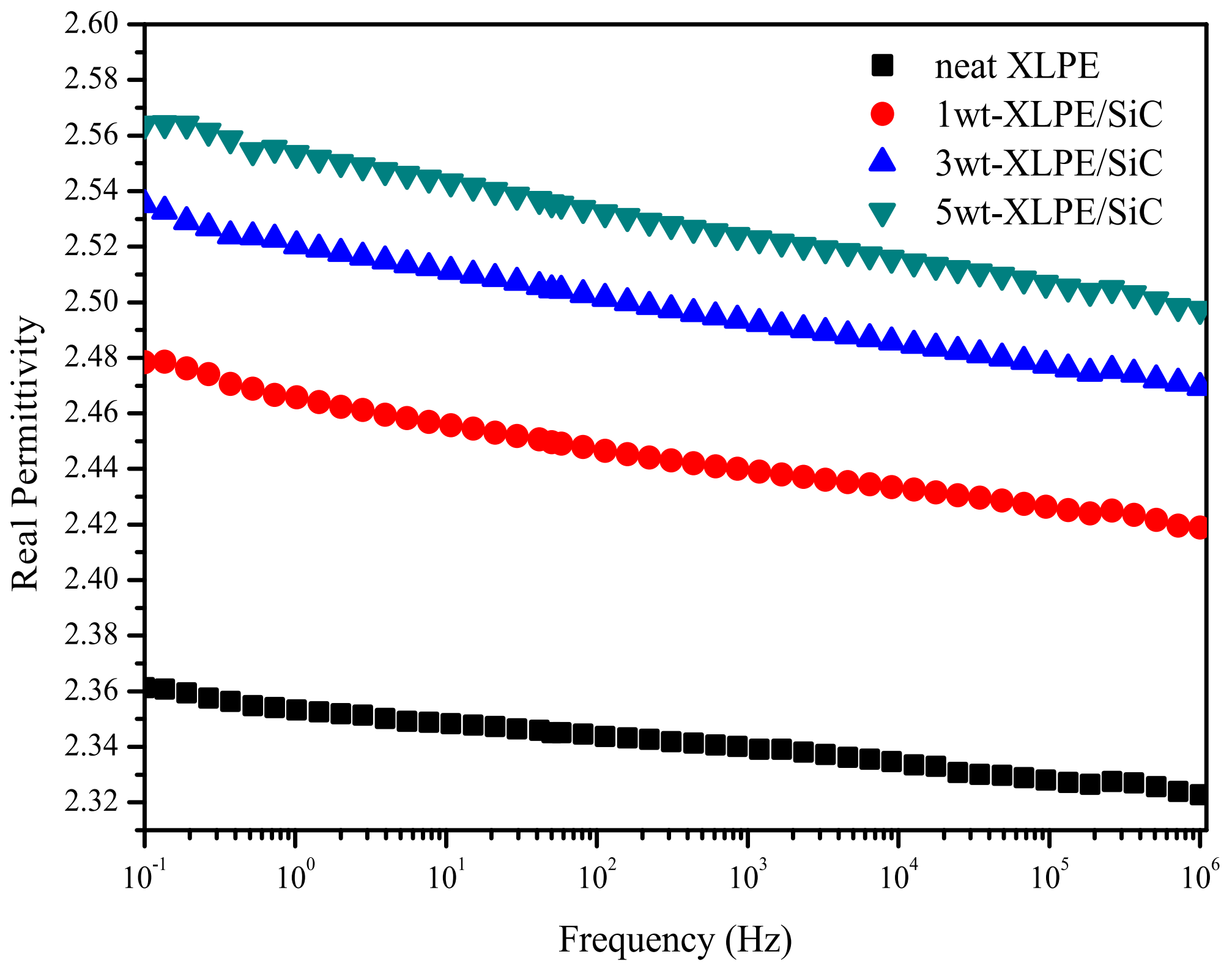




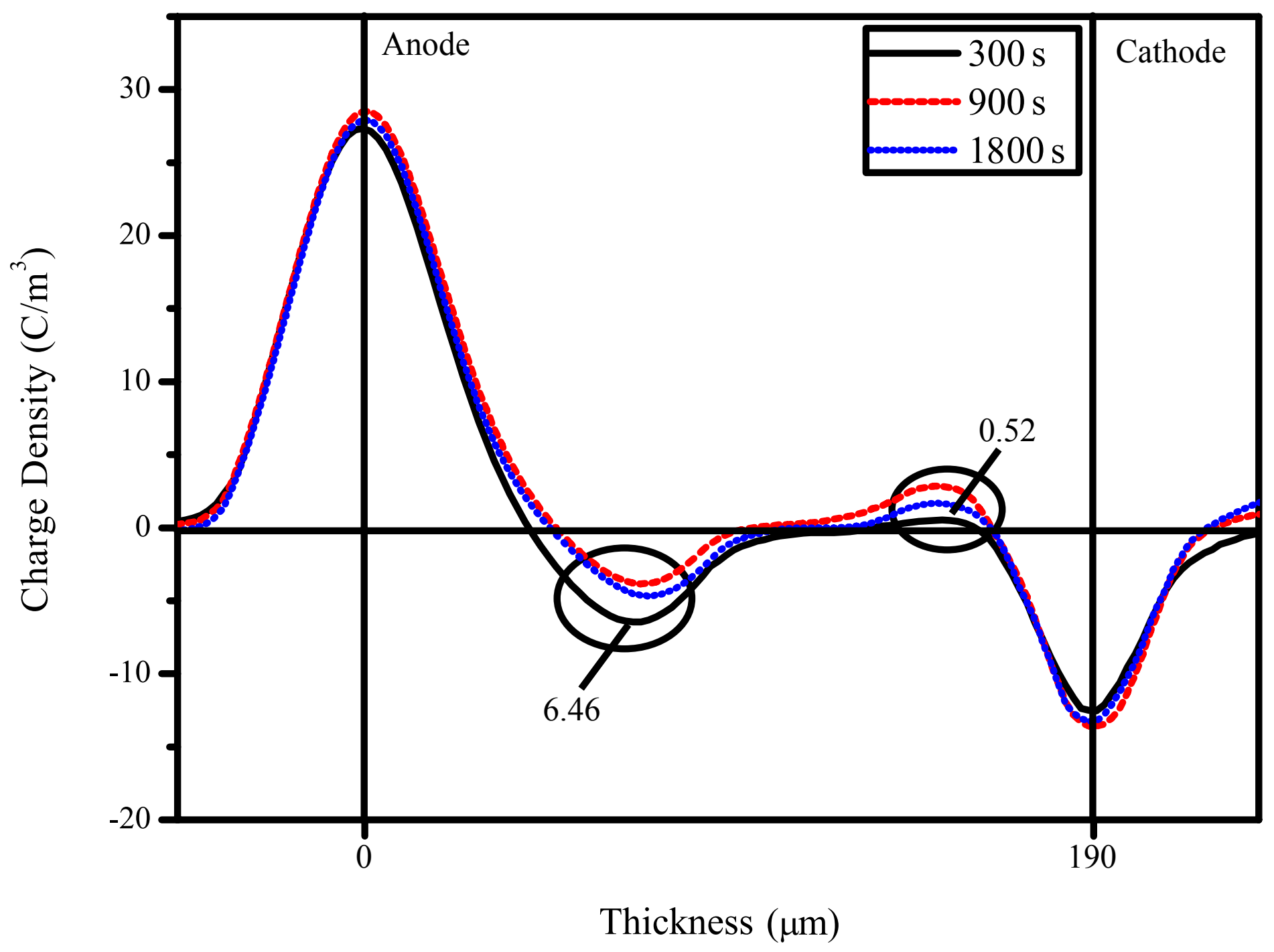




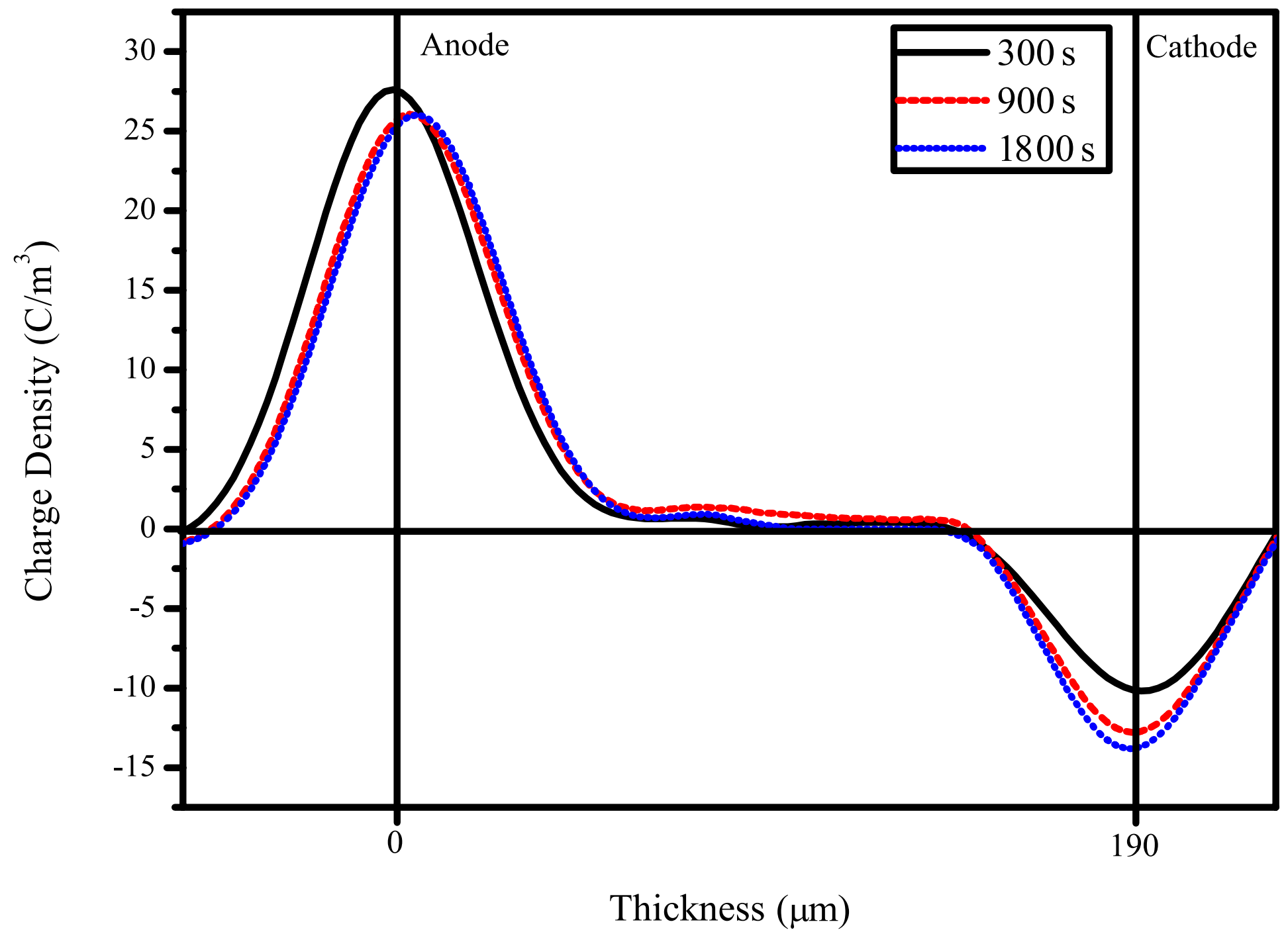




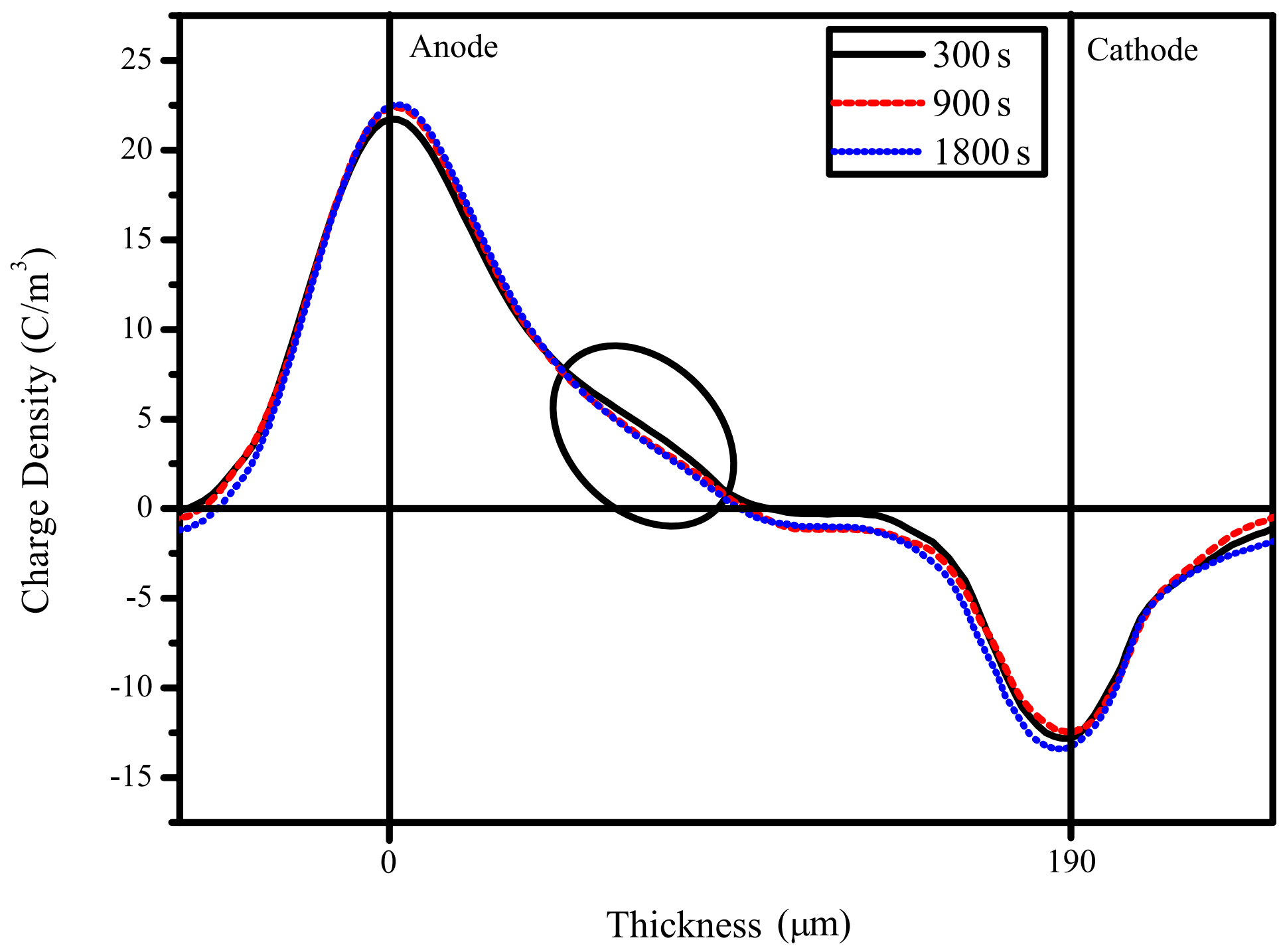




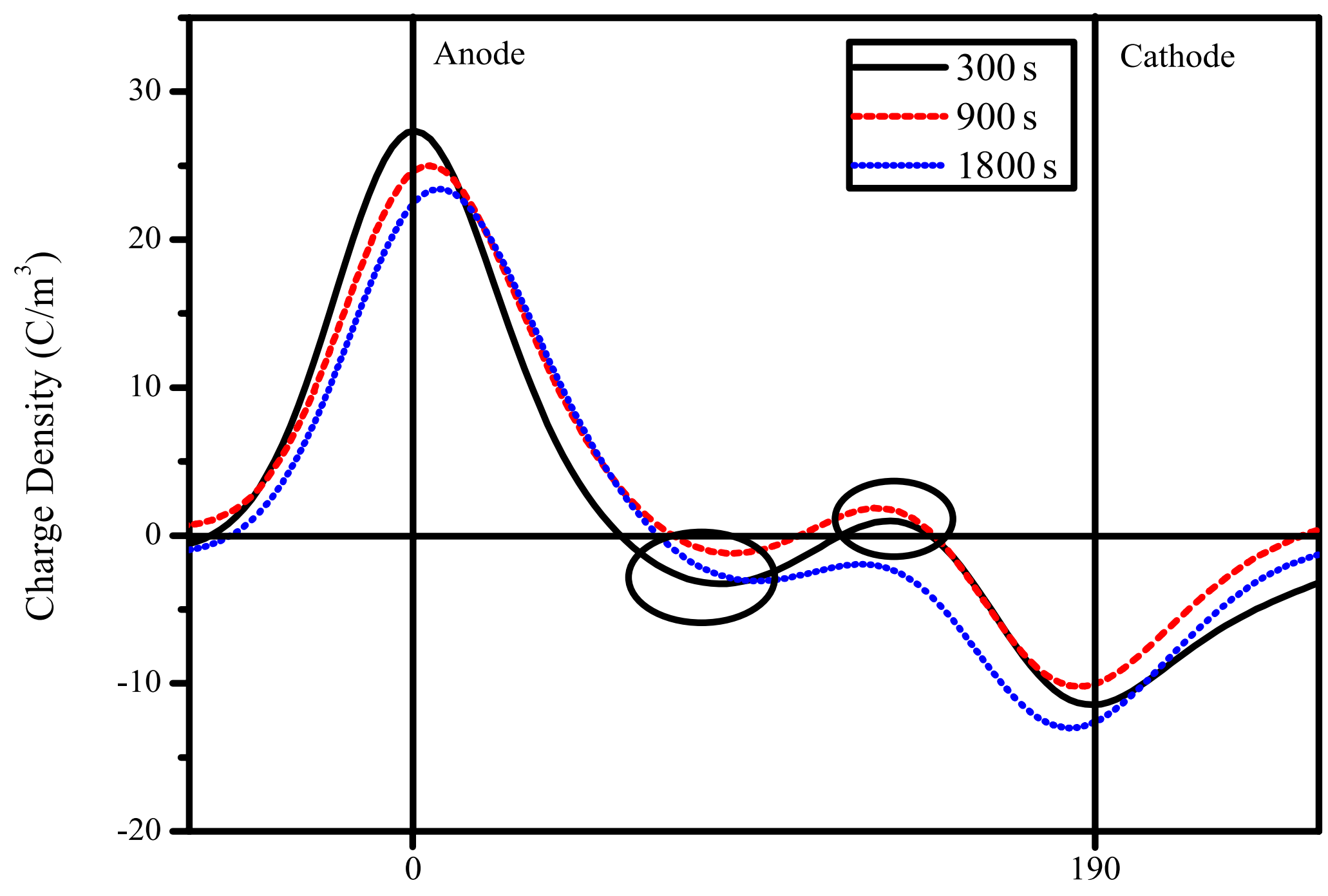

Thickness $(\mu \mathrm{m})$ 


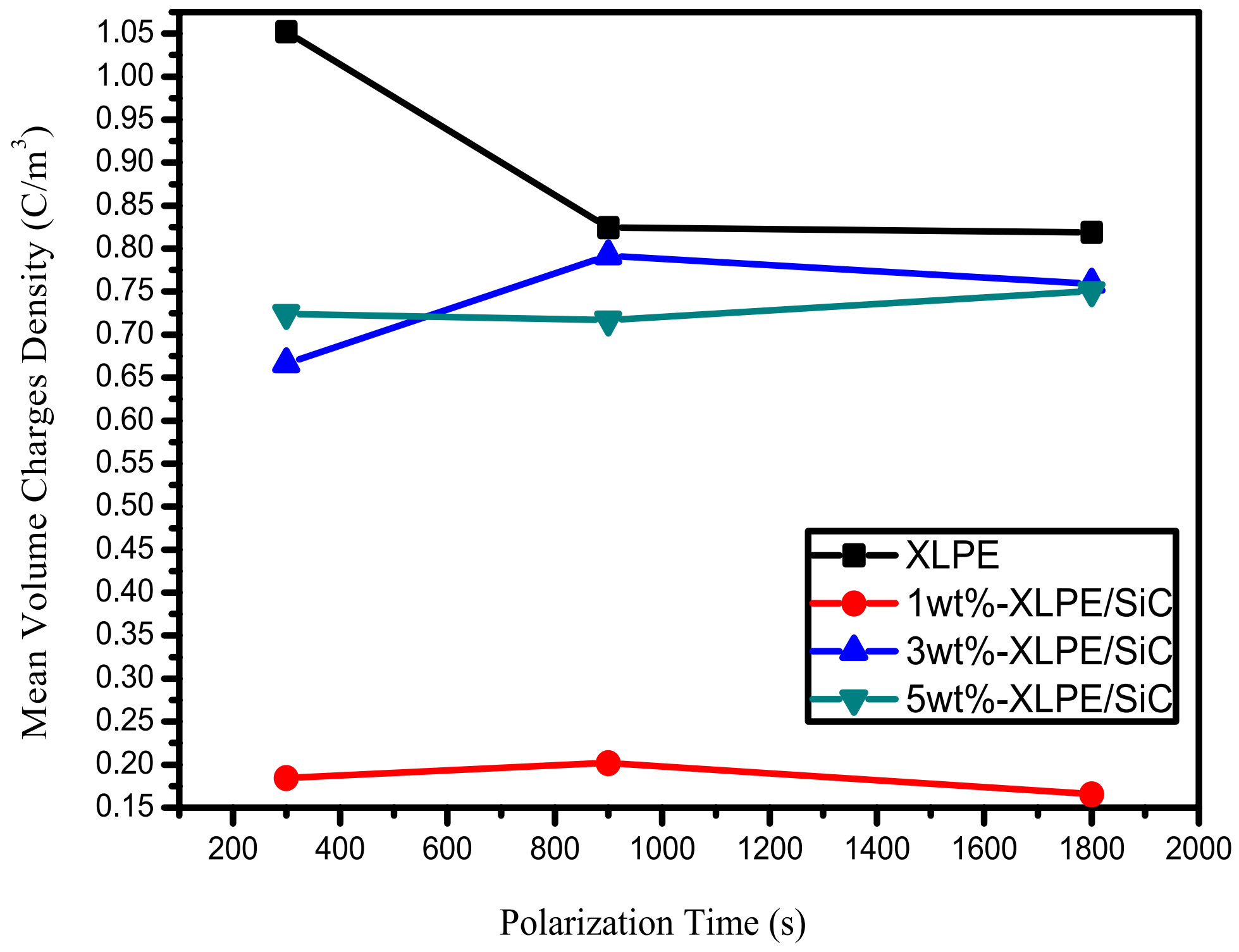




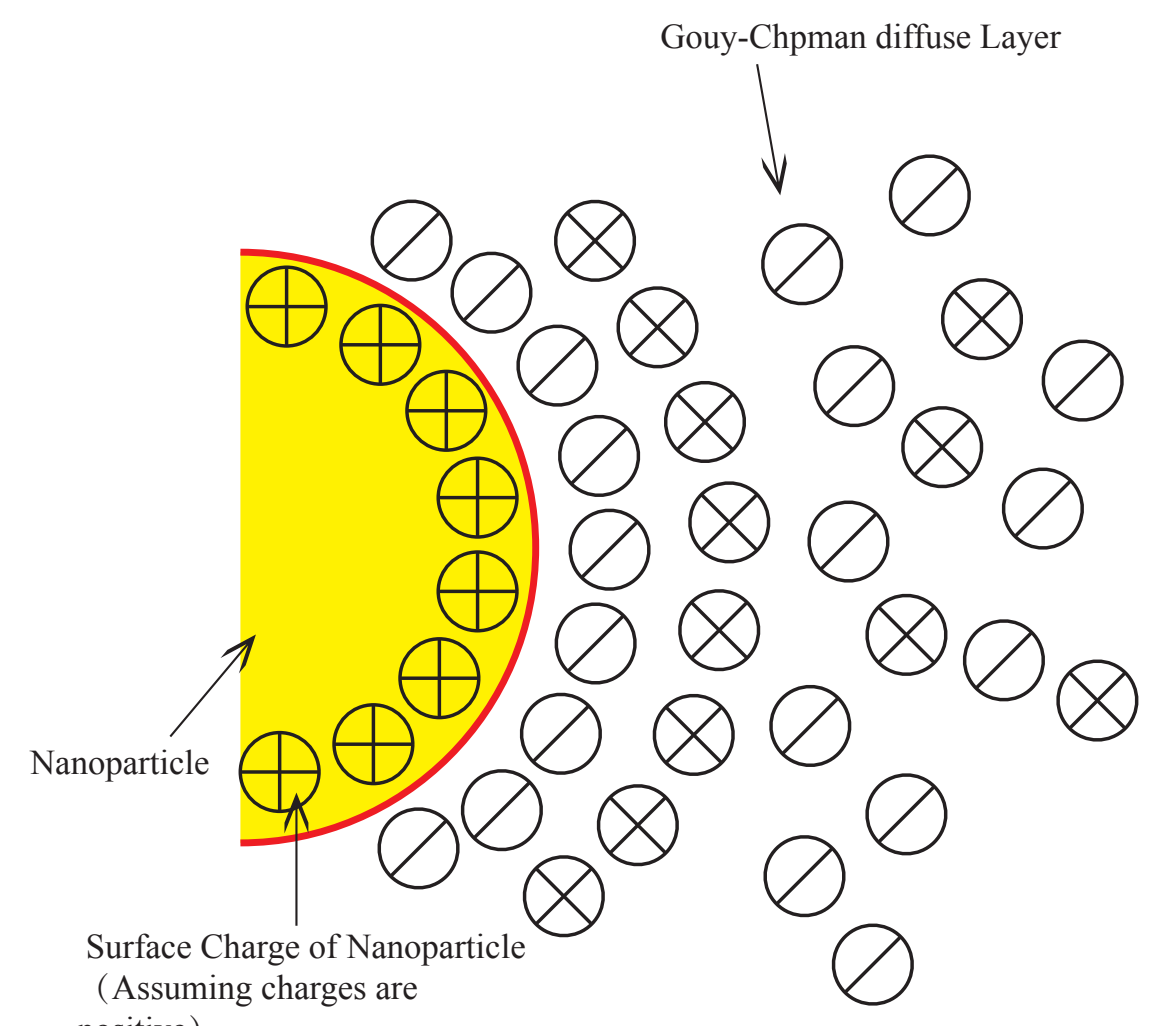
positive) 


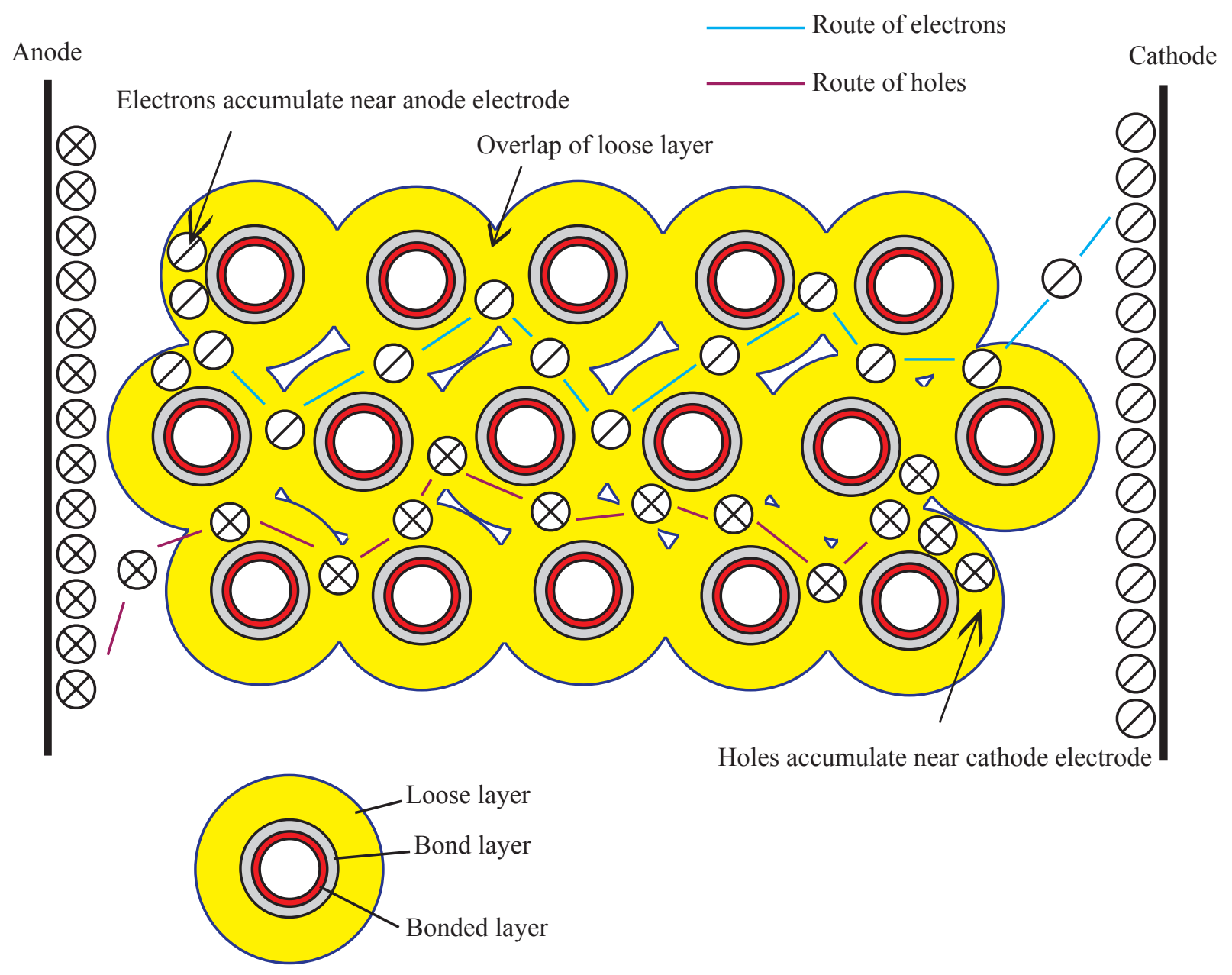

Multi-core model 